\title{
Iliopsoas abscess due to methicillin resistant staphylococcus aureus in a 26 day old neonate
}

\author{
*H K K T Duminda ${ }^{1}$, A K Lamahewage ${ }^{2}$, A Liyange ${ }^{3}$, D M H Fernando ${ }^{4}$
}

Sri Lanka Journal of Child Health, 2015: 44(3): 167-168

(Key words: Iliopsoas abscess, MRSA, neonate)

\section{Case report}

A 26 day old baby girl, delivered by normal vaginal delivery at term, with a birth weight of $3.4 \mathrm{~kg}$, presented with swollen left thigh, reduced movements of the left leg and fever for 3 days. She had normal feeding. There was no maternal fever or contact history of tuberculosis (TB). She had uneventful prenatal and immediate postnatal periods.

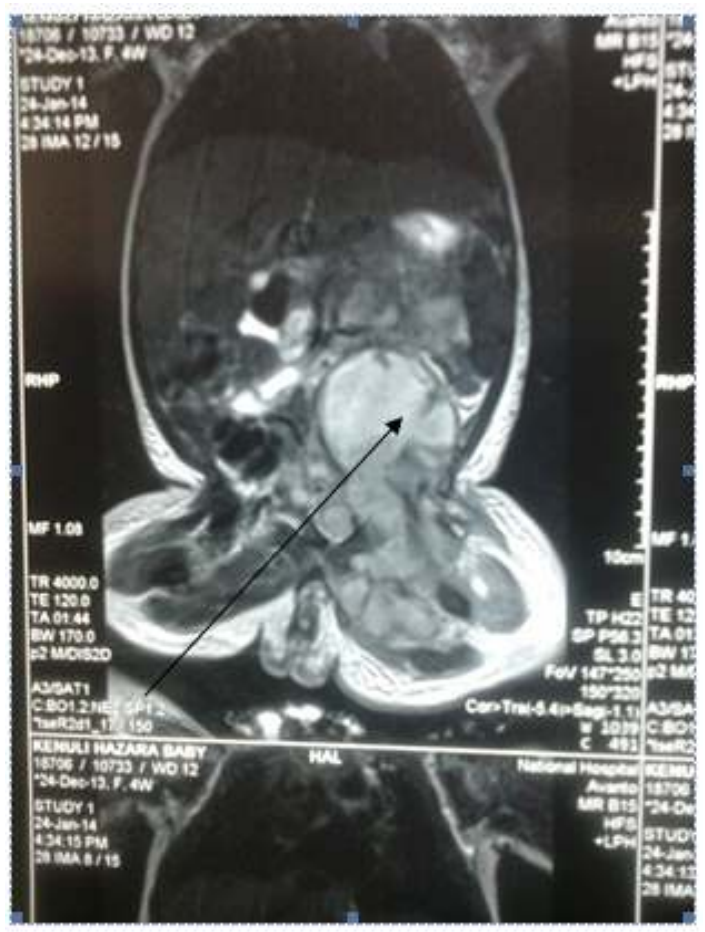

Figure 1: Coronal MRI of abdomen showing left psoas abscess with extension to thigh

${ }^{1}$ Registrar, ${ }^{2}$ Consultant Paediatric Surgeon, ${ }^{3}$ Senior Registrar, Lady Ridgeway Children's Hospital, Colombo

*Correspondence: thilakduminda@yahoo.com

(Received on 23 March 2014: Accepted after revision on 30 May 2014)
On examination, child was febrile $\left(101^{0} \mathrm{~F}\right)$ with no skin sepsis and stable vital parameters. There was a palpable non mobile lump in left iliac fossa. Left thigh was swollen diffusely up to mid-thigh with no definite mass. Ultrasound scan (USS) revealed left iliopsoas abscess (IPA) with inconclusive extent. Magnetic resonance imaging (MRI) and computed tomography (CT) scans revealed a multiloculated abscess extending into upper thigh (Figure 1).

White blood cell (WBC) count was 19,300/cu mm with 54\% neutrophils and 40\% lymphocytes. Creactive protein (CRP) was $144.2 \mathrm{mg} / \mathrm{L}$ and blood cultures were negative. Surgical exploration was done through a left Rutherford Morrison incision approached extraperitonealy. Large abscess drained $50 \mathrm{ml}$ of thick pus and specimens were taken for bacterial culture. Abscess cavity extended to the thigh under the inguinal ligament. Corrugated rubber drain was kept for five days. Culture revealed methicillin resistant staphylococcus aureus (MRSA) but other cultures and PCR for TB were negative. Patient was given intravenous amikacin and vancomycin for two weeks with good response.

\section{Discussion}

IPA is extremely rare in neonates ${ }^{1}$. It is primary or secondary and can be overlooked easily ${ }^{1}$. If not treated in time it is invariably fatal but is otherwise potentially curable ${ }^{1}$. The management is aided by isolated cases reports of which there are only 18 up to date in the English literature ${ }^{1}$. Unlike in adults, secondary IPA is rare children ${ }^{2}$. IPA develops towards the second half of the neonatal period rather than the immediate postnatal period ${ }^{1}$. Most reported cases of IPA presented with a triad of symptoms: femoral triangle swelling, reduced mobility of lower limb, and mild fever ${ }^{1}$. Some had a palpable abdominal mass or a mass in the femoral triangle but this was not commonly encountered ${ }^{1}$. WBC counts were in the range of $20,000-30,000 / \mathrm{cu} \mathrm{mm}^{1}$.Only a few cases presented with high fever $\left(>38^{\circ} \mathrm{C}\right)^{1}$. 
The common differential diagnosis is septic arthritis of the hip joint. This neonate developed swelling of the groin and femoral region with reduced movements of the left leg. X-ray of left hip joint and thigh was normal except for significant soft tissue swelling of the proximal half of the thigh. Ultrasonography is the first line modality used for diagnosis ${ }^{1}$. USS done without sedation or anaesthesia usually suggests the diagnosis ${ }^{1}$. Drawbacks of USS are not demarcating the extent of the abscess and operator dependency ${ }^{1}$. CT scan and MRI are useful to obtain more detailed information of the anatomical extent of the abscess and status of the hip joint and lumbar vertebrae ${ }^{3}$.

In this neonate, $\mathrm{CT}$ and MRI scans showed extension of the abscess into the thigh and the presence of multiloculi precluded USS guided drainage. Therefore when a newborn presents with poor mobility of a lower limb, USS and CT or MRI are warranted to check the retroperitoneum and even in the absence of high fever there is a need for a high degree of suspicion for timely diagnosis. Most neonatal causes of IPA are not detected. Identified sources are: bacteraemia, discitis (T12-L1 vertebrae $)^{4}$, septic arthritis of hip joint and skin pustules. One neonate had leukocyte adhesion defect $^{2}$. IPA probably occurs as a result of haematogenous spread from an occult source in the body because of weak immunity. That is supported by the fact that Staphylococcus aureus as commensal flora of the skin is the most frequently isolated organism from $\mathrm{IPA}^{3}$. A few cases involved MRSA, Klebsiella pneumoniae and Streptococcus pneumoniae $^{1}$. It is possible that the short delay between birth and onset is attributable to these causative organisms, especially MRSA.

With respect to treatment, most were given intravenous antibiotic therapy empirically because of high WBC count and CRP and delay in diagnosis ${ }^{1}$. Antibiotic alone was often insufficient ${ }^{1}$. Most abscesses were multiloculated and therefore managed by open surgical drainage. Extra-peritoneal approach was preferred ${ }^{4}$. In a few neonates, the abscesses were diminished with antibiotic therapy alone ${ }^{5}$. USSguided drainage has recently been reported as a minimally invasive method of drainage but precluded in the index patient as the abscess was multiloculated $^{6}$. Superiority of percutaeneus USS guided drainage over open drainage is controversial in neonates but it is not the sole therapy in reported adult case series and most required open drainage subsequently because of inadequate drainage due to high viscosity of the pus ${ }^{5}$. Duration of antibiotics depend on clinical progression which is usually 2 weeks in most reports ${ }^{1}$. In one neonate the abscess drained through an inguinal incision alone ${ }^{1}$. In follow-up CRP and serial USS are used. Despite significantly high inflammatory markers (WBC and $\mathrm{CRP}$ ) recurrence after drainage and septicaemia were quite low ${ }^{1}$. The prognosis was good in most of reported cases without residual effects ${ }^{1}$.

\section{References}

1. Atsushi H, Kazuhiro K, Jun K, Yuji W, Sanae K, Hisamichi et al. Neonatal iliopsoas abscess. Pediatrics International 2012; 54(5):712-4. http://dx.doi.org/10.1111/j.1442200X.2012.03593.x PMid: 23005905

2. Nalan K, Ozden T, Cigdem A, Nevin H, Arzu H, Erdal A. Iliopsoas abscess in the neonate with immunodeficiency. Pediatrics International 2012; 54: 439-40.

http://dx.doi.org/10.1111/j.1442200X.2012.03623.x PMid: 22631579

3. Zych GA, McCollough NC. Acute psoas abscess in a newborn infant. Journal of Pediatric Orthopedics 1985; 5: 89-91. http://dx.doi.org/10.1097/01241398-19850100000016

PMid: 3980715

4. Rakesh M, Sumantra S. Spondylodiscitis with primary psoas abscess in a neonate. Indian Pediatrics 2012; 49: 681 .

5. Tabrizian $\mathrm{P}$, Nguyen $\mathrm{SQ}$, Greenstein A, Rajhbeharrysingh U, Divino CM. Management and treatment of iliopsoas abscess. Archives of Surgery 2009; 144: 946-9.

http://dx.doi.org/10.1001/archsurg.2009.144 PMid: 19841363

6. Dib M, Bedu A, Garel C, Mazda K, PhilippeChomette P, Rajguru M, et al. Ilio-psoas abscess in neonates: treatment by ultrasoundguided percutaneous drainage. Pediatric Radiology 2000; 30(10):677-80. http://dx.doi.org/10.1007/s002470000309 PMid: 11075599 